\title{
Spectral Narrowing and Brightness Increase in High Power Laser Diode Arrays
}

\author{
Niklaus Ursus Wetter \\ Centro de Lasers e Aplicações, \\ Instituto de Pesquisas Energéticas e Nucleares de São Paulo, \\ Brazil
}

\section{Introduction}

Diode laser arrays, also called diode bars, are very important light sources that are generally used for pumping of other solid-state lasers and in medical and industrial applications that require high power but low intensity. Although they are very interesting from a commercial point of view, they did not find their way into many important applications because of their very low beam quality and spectral brightness.

This book chapter will explain why diodes have such a low spectral and spatial beam quality and give an overview on the different techniques to improve it.

In recent years, technology has achieved advances that permit to compare the spatial and spectral brightness of diode lasers to that of crystal solid-state lasers. Recent results of the literature are spatial brightness of $10^{2} \mathrm{MW} / \mathrm{cm}^{2} \mathrm{str}$ with a beam quality product of $\mathrm{M}_{x}^{2} \mathrm{M}_{y}^{2}=$ 20 and narrowing of a diode-arrays' emission bandwidth to the $\mathrm{GHz}$ or even $\mathrm{MHz}$ range.

Diode laser arrays are nowadays the basic building blocks of high power semiconductor lasers. They can continuously emit up to approximately $100 \mathrm{~W}$ at specific visible to near infrared wavelengths and can be stacked to give even much higher output powers of the order of several thousand watts. They find their main applications in materials processing, medical applications, printing and optical pumping of solid state lasers. Amongst the many industrial processing applications are welding, hardening, brazing and sintering. Medical and dental applications for diode bars include surgery, hair- and tattoo removal, photodynamic therapy and tooth whitening. Diode bars are also widely used in the military for targeting applications and therefore the distribution of some high power diode arrays is controlled.

Diode arrays present small size, efficiency in excess of $50 \%$, directly convert electricity into light and are the cheapest lasers in terms of watts per dollar on the market. For these reasons it would be of great benefit for commercial and scientific applications if they could substitute the common and expensive gas and crystal lasers that use resonators which are cumbersome to align and generally require highly skilled assembly and maintenance personnel. The reason that diode arrays so far did not substitute gas and crystal lasers in almost all applications lies in their low spectral and spatial beam quality. 
A single diode bar is a linear array of approximately 19-70 individual diode emitters. The overall emitting area of a diode bar has generally a width of $1 \mathrm{~cm}$ and is $1 \mu \mathrm{m}$ high. Due to the small height, comparable to the emission wavelength, the emitted beam diverges strongly in this direction (fast divergence axis) whereas in the other direction, given by the array of emitters, the divergence is smaller (slow divergence axis). As a result, the combined output beam is almost diffraction limited in the fast direction but has divergence that is more than thousand times that of a diffraction limited beam in the other direction.

This very poor beam quality hampers the usefulness of diode bars in many applications. Specifically, when trying to focus the beam tightly, very different waist sizes, waist positions and depth of foci are obtained for the fast and slow axis, making it almost impossible to tightly focus the diode's beam and making them resemble more flash light lamps than collimated laser beams.

Another reason that hinders the usefulness of diode beams is their spatial characteristics. From an application point of view it is in most cases interesting that the emission frequency is stable and as narrow as possible. Applications include pumping of solid-state lasers and laser cooling of gases for atomic clocks. Diodes not only change their emission wavelength strongly as a function of temperature but they also show linewidth broadening as a function of pump current. Typical linewidth are of the order of several nanometers and changes with temperature are around $0.25 \mathrm{~nm} /{ }^{\circ} \mathrm{C}$.

\section{Diode bar fabrication and design}

The main difference between a semiconductor laser and a conventional solid state laser is that the diode laser converts directly electric power into light without the need of an optical pump. It therefore immediately achieves much higher wall-plug efficiency than any other solid-state laser (around 50\% wall-plug efficiency, although more than $70 \%$ have been achieved at room temperature (Knigge et al., 2005)). That combined with its ability to be easily fabricated in mass production makes the semiconductor laser the cheapest laser per Watt of output power and the preferred laser candidate whenever its beam and spectral characteristics are apt for the desired application.

The constructive basics of a simple laser diode are well known. A semiconductor diode laser, or simply laser diode, is formed by doping thin layers on a direct band-gap semiconductor crystal wafer to produce n-type and p-type regions on top of each other, resulting in a p-n junction. The favored crystal growth techniques for semiconductors are liquid phase epitaxy and chemical vapor deposition using metal-organic reagents (MOCVD) but also molecular beam epitaxy (MBE) is frequently used. Forward electric bias across the junction forces holes and electrons to enter the depletion region (a region void of carriers that forms close to the junction because of the difference in electric potential between the ntype and p-type semiconductor). There, the carriers recombine generating preferentially spontaneous emission.

However, in order to achieve better efficiency than with this simple model, the commonly preferred constructive method since the 1990s is the separate confinement heterostructure (SCH) quantum well laser diode. This laser has a quantum well layer at the junction center made of high index material that serves to concentrate electrons in energy states that are 
prone to the emission of light. Additionally, the quantum well is flanked by two layers of different p-type materials on one side and two layers of n-type materials on the other side. As one moves farther away from the junction, each layer is made of a material with less high refractive index in order to confine the light generated by the recombination process into a very thin layer around the junction called active area. The refractive index graduation can be achieved for example by co-doping the GaAs semiconductor with small amounts of aluminum (wavelength range $630 \mathrm{~nm}$ to $1 \mu \mathrm{m}$ ). Typical dimensions for these layers are 0.01 $\mu \mathrm{m}$ for the quantum well layer and $1 \mu \mathrm{m}$ for the layers of high index p-type and n-type materials closest to the quantum well. Due to the small dimension of the active area perpendicularly to the junction, all semiconductor diodes are essentially single transverse mode in that direction.

As with other lasers, laser action is achieved by giving a preferred direction to the emission of light generated in the active region and by building a resonator. In a laser diode this is generally done by fabricating the metal contact on top of the diode in the form of a long and thin ribbon. Recombination occurs within the semiconductor underneath this contact and preferential emission is achieved in the strip's direction, much as with a solid-state laser rod. Next, the semiconductor crystal is cleaved at its two ends to form a Fabry-Perot resonator. Depending on desired gain and spectral characteristics of the laser diode the end-facets maybe anti-reflection coated or uncoated, in which case they have a reflectance of approximately $30 \%$ in the visible region. The width of the active region parallel to the junction is generally of several microns and the length is approximately one millimeter.

Due to the small height of the active region, the stimulated emission perpendicular to the layers is strongly diffracted at the exit facet into an angle of approximately 25 degrees (FWHM) with Gaussian beam quality (fast axis direction). Parallel to the layers, the beam quality depends on the width of the electric contact strip and on the number of strips whereas the divergence angle remains generally around 10 degrees (slow axis direction). The fast axis divergence angle is always larger than in the slow direction because of its smaller dimension and also because of the larger index variation along the fast axis direction which causes larger numerical aperture (NA). Very small strips (width of $2-7 \mu \mathrm{m}$ ) lead to single transverse mode with overall diffraction limited beam quality and elliptical beam proportions but have also small power, not suitable for high power beam applications. Furthermore these devices can be made single frequency by limiting the longitudinal modes using suitable wavelength selective devices such as gratings that can be incorporated on chip or externally to the diode. The maximum output power for these devices is around a couple of hundreds of milliwatts for commercial devices although close to one watt has been achieved (Wenzel et al., 2008). Further power enhancement is obtained using an amplifier at the end of the diode in the form of a MOPA (master oscillator and power amplifier), which is a series of more or less independent devices. But also tapered amplifiers are common that employ the whole device including amplifier as resonator. The latter device achieved recently single transverse mode (TEM $\mathrm{T}_{00}$ ) output powers in excess of $10 \mathrm{~W}$ at slightly reduced beam quality, but such devices are mostly only experimental (Muller et al., 2011).

Single mode diode lasers have the highest spectral and spatial beam brightness. If higher powers are required the fabrication technology goes different ways and one has to decide what is more important, spatial or spectral brightness or a combination of both. 
Brightness $B$ is defined as:

$$
B=\frac{P(\text { watt })}{A\left(\mathrm{~cm}^{2}\right) \cdot \Omega(\text { sterad })}=\frac{P}{w_{x} w_{y} \theta_{x} \theta_{y} \pi^{2}}=\frac{P}{\lambda^{2} M_{x}^{2} M_{y}^{2}}
$$

Where $P$ is the total power, $A$ is the area and $\Omega$ and $\theta$ are the solid angle and half angle divergence, respectively, at $1 / \mathrm{e}^{2}$ of the peak power. $\lambda$ is the wavelength and $M^{2}$ is the beam quality factor, which is 1 for Gaussian beams and gets bigger as quality decreases. Spectral brightness is defined as the brightness divided by the diodes bandwidth.

As the contact strip is made wider the output power increases and with it the locally generated heat (about $50 \%$ of the consumed energy) whereas beam quality degrades. $20 \mathrm{~W}$ of output power have been achieved at $980 \mathrm{~nm}$ emission wavelength in $100 \mu \mathrm{m}$ wide strip (Crump et al., 2009). These devices that go by the name of "broad area" diodes (BAL) are commercially available and have output powers of up to more than ten watts with beam quality factors in the directions parallel and perpendicular to the junction of typically 20 and 1 , respectively. The width of the broad area can be increased and $500 \mu \mathrm{m}$ is common but without considerable increase in output power and at a considerable loss of beam quality ( $\mathrm{M}^{2}$ parallel to the junction of about 100).

At even larger strip width the transverse direction can develop transverse lasing, amplified spontaneous emission (ASE) or even filamentation. These parasitic phenomena do not only cause losses but also dynamical instabilities and inhomogeneities in the beam profile. It is therefore more convenient to break the active region into a series of parallel strips. The drawback is a strong decrease in beam brightness that is proportional to the amount of "dead space" that is introduced in between the adjacent strips.

Generally there are 19 or 24 emitters in the case of continuous (cw) diode lasers and up to 60 for quasi-continuous (qcw) diode lasers. This high power diode laser (HPDL), which has total dimensions of approximately 1 millimeter length, $10 \mathrm{~mm}$ width and about $0.1 \mathrm{~mm}$ height is called a "diode array" or "diode bar". Individual emitter width is generally $100 \mu \mathrm{m}$ to $200 \mu \mathrm{m}$ and emitter spacing is $500 \mu \mathrm{m}$ or $400 \mu \mathrm{m}$ for the 19 and 24 emitter diode bars, respectively, with fill factors of $20 \%$ to $30 \%$. The spacing between emitters, which is mainly dictated by cooling requirements, can be made much smaller for qcw diode bars reaching fill factors of up to $90 \%$. Maximum output power for commercial cw diode bars is above 100 $\mathrm{W}$ and for qcw diode bars more than $300 \mathrm{~W}$ at less than $500 \mu$ s pulse duration and less than $10 \%$ duty cycle can be achieved. Above values are for conduction cooled diodes. For water cooling the heat removal is more efficient and gets even better with micro-channel water cooling which permits up to $80 \%$ fill factor in continuously operated diode bars. As can already be seen and will be shown also further ahead, efficient heat removal is a key factor in obtaining higher brightness for the same amount of output power. Several groups have reported in excess of $700 \mathrm{~W} \mathrm{cW}$ per bar with production prototypes mainly by increasing the cooling efficiency using different means such as extending the emitter length from $1 \mathrm{~mm}$ to more than $3 \mathrm{~mm}$. In all cases the beam quality perpendicular to the junction is close to diffraction limited whereas parallel to the junction the beam quality parameter $\mathrm{M}^{2}$ is well in excess of 1000. At the junction exit the beam is a linear array of individual beamlets that overlap after a couple of millimeters. In the far field this elliptical beams' divergence angles are 30 degrees along the fast axis and 10-12 degrees along the slow axis. 
Output power scales with fill factor in part because the maximum power density inside a diode bar is limited by the onset of catastrophic failure on the diodes exit facet. This failure is due to residual absorption at the beam exit facet caused by surface states at the cleavage plane that do not exist within the bulk. This problem hinders the maximum achievable power particularly in GaAs-based semiconductors and a great deal of effort has been spent in recent years to overcome this residual absorption by "passivation" of the exit facets.

The diode bar is normally soldered onto a thin submount which also serves as electrical contact. This submount is then attached to a large heatsink for effective removal of the heat. During this process the very thin $(0.1 \mathrm{~mm})$ but large $(1 \mathrm{~cm})$ diode array is easily bend by the pressure applied to bond it to the submount and heatsink but also by the differential expansion during the solder process. The overall effect of this process causes a curvature of the array called "smile" which amounts to about $1-10 \mu \mathrm{m}$ difference in height at the center of the bar with respect to the bar ends. Some manufactures also bond the bar directly to the copper heat-sink, which is referred to as "direct bonding" and causes generally less smile.

The problem with array curvature is that it degrades the beam brightness. Once the diode is manufactured, the bar curvature is fixed. Therefore it is of importance to understand the constructive details of diode bar manufacturing to apply effective countermeasures. The deviation from linearity of the smile makes it impossible for a single collimation lens in front of the emitters to be perfectly positioned for all emitters along the bar. This first lens, which serves to collimate the fast-axis (FAC lens), is attached directly in front of the diode bar and is generally a plano-aspherical cylindrical lens for low aberration that resembles approximately a $1 \mathrm{~cm}$ long piece of fiber. The focal length of this type of lenses varies from 0.3 to $1.5 \mathrm{~mm}$ and the numerical aperture is high, usually between 0.5 and 0.8 to accommodate the strong divergence in the fast axis direction. After the FAC lens, the beam resembles a sheet of light with $1 \mathrm{~cm}$ width and of approximately one millimeter height. In the far field the divergence angles are 4-6 degrees along the fast axis and continue 12 degrees along the slow axis.

After attaching the collimation optics to the diode bar the beam errors are set and the rays emitted by the diode occupy a larger volume than the theoretical minimum. But the errors can be fixed if it is possible to design and introduce a suitable optical element or optical system.

\subsection{Diode bar beam quality assessment}

The beam errors increase the difficulty to design the subsequent optics and reduce the efficiency of the whole optical system in terms of spatial and spectral brightness. It is therefore of great importance to assess the specific nature of these imperfections in order to design effective countermeasures that can in many cases completely eliminate the consequences of these errors. The normal assessment technique for the beam quality of any laser is by means of measuring the quality parameter $\mathrm{M}^{2}$. This is a simple measurement that can give data on beam divergence, focal spot size and confocal parameter but it is not enough information to characterize which is the appropriate correction technique in the case of beam errors and more elaborate beam assessment methods have to be applied. 


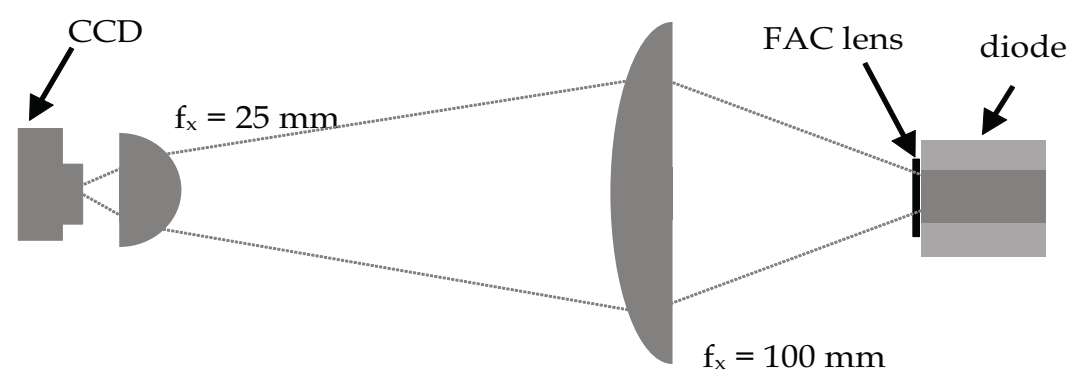

Fig. 1. Simple imaging system to visualize the beam errors introduced by the diode and the FAC lens: the slow $x$-axis direction is collimated by two cylindrical lenses.

Although there are nowadays many assessment techniques available, one can obtain most required information by using a simple imaging system that projects the individual beamlets onto a CCD camera (Wetter, 2001). If the diode bar has a FAC lens incorporated and therefore has its fast axis already collimated, this system may comprise simply two cylindrical lenses to image the slow axis direction.

Imprecision in the alignment and positioning of the FAC lens at the micron level further reduces the brightness. Failure to align the lens parallel to the junction causes "skew" that projects the individual beamlets from the emitters at different heights. The problem with skew is that it causes astigmatism and coma as shown in figure 2. Another common problem is to keep the precise distance of the fiber to the array along the whole width of $1 \mathrm{~cm}$. This relative focus error manifests itself in different spot sizes of the beamlets at the image or focus plane, further increasing astigmatism and decreasing the brightness. Earlier FAC lenses were simple glass fibers attached in front of the bar. These fiber-FAC lenses could easily be bent and introduce further curvature into the highly astigmatic exit beam. Nowadays they have mostly been replaced by bigger glass lenses that have additional support structure to decrease the possibility of bending.
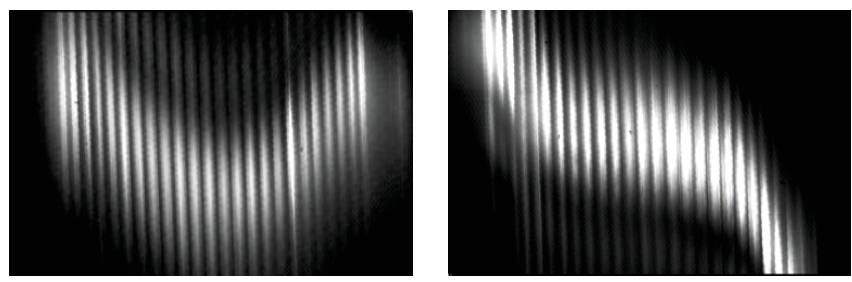

Fig. 2. Two beam errors that are encountered with diode bars; from left to right: smile and skew. The images have been taken with the system in figure 1.

\section{Beam-shaping techniques}

Beam-shaping techniques applied to diode arrays have two purposes. The first goal is to circularize the highly astigmatic beam of uneven beam quality factors in such a way that it can be used in most practical applications that require generally focusing or collimation and round spot sizes. The second goal is to increase brightness. Brightness increase augments 
local absorption at the samples surface and inside the sample which leads to higher temperatures and therefore to more efficient material removal and faster processing speeds. As a result, it increases the range of applications the diode bars can be used for. For instance in medical application, low brightness is enough for hair removal whereas high brightness is necessary for surgery. Both applications may use a laser system composed of the same diode bar component. The difference being that the later application requires additional beam shaping with post-bar additional optical components inside the beam path.

Most practical beam-shaping techniques used for beam circularization are based on methods that geometrically transform the diode radiation into a rounder spot size. It is convenient for this purpose that the diodes beam consists of individual beamlets which can be rearranged by suitable optical devices. One of the first and still used methods of beamshaping consists of individual fibers, only slightly larger than the individual emitter width, which are precisely positioned in front of each of the diode bar's emitters at a very short distance. The fibers can then be bundled into the desired geometrical form which is for most applications of circular shape. Many other beam-shaping systems consist of optical components that chop the large beam coming from the diode bar into a number of subbeams. These sub-beams are then reflected, diffracted or refracted by clever positioned mirrors, prisms, gratings and micro-lenses in such a way that the sub-beams are stacked on top of each other in order to generate a round spot size.

In figure 3 one can see such a beam-shaper composed of two plane parallel mirrors that are inclined sideways (around a vertical y-axis) and backwards (around the horizontal x-axis). The incoming light sheet from the diode bar passes just above the first mirror where one fifth of the beam is cut-off by the second mirror passing on directly whilst four fifths get reflected slightly downward and to the left (negative $x$-axis direction) back onto the first mirror. This scheme is repeated until all five parts emerge from the two mirror beam shaper, stacked on top of each other and having ideally a square shape and equal quality factors in the $\mathrm{x}$ - and $\mathrm{y}$-directions.

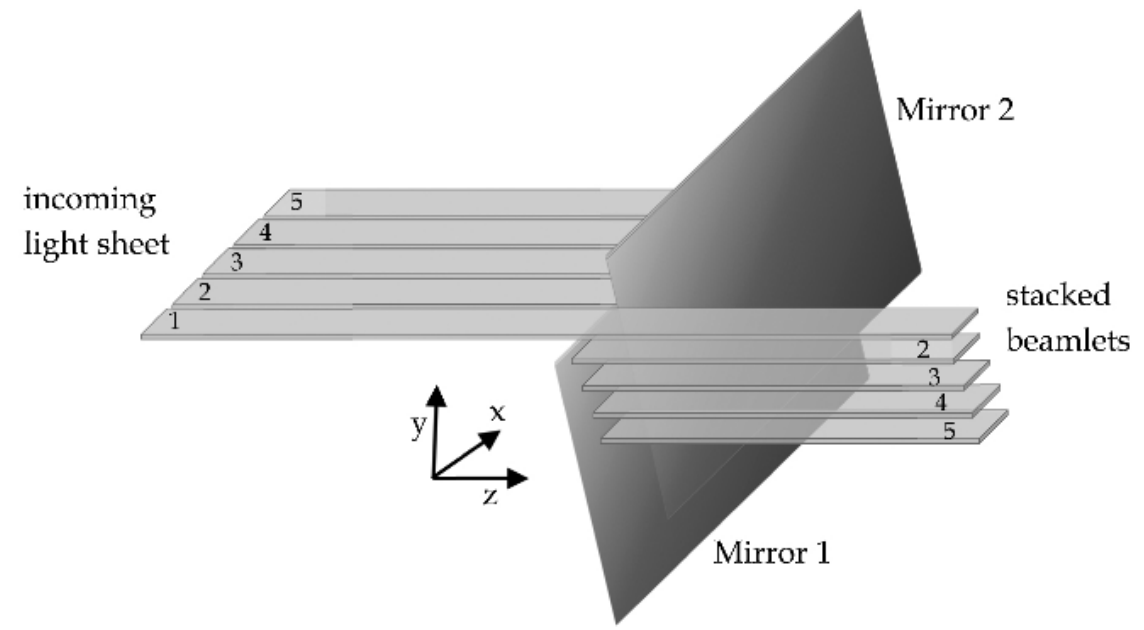

Fig. 3. Two mirror beam shaper used to cut a homogenous light sheet into five sub-beams that are stacked on top of each other. The goal is to achieve a new beam with equal dimensions and quality factors in the horizontal and vertical directions. 


\subsection{Correction of diode beam errors}

Most beam-shaping techniques depend upon a good quality light sheet being emitted by the bar. For instance, if the diode shows smile as in figure 2, the middle part of the beam will get clipped by mirror 1 in figure 3 upon the first passage of the light sheet above the mirror. This is not only an issue for the two-mirror beam shaper but almost all beam-shaping methods rely on good alignment of the individual beamlets in order to reconfigure the beam without losses. In these cases skew, smile and relative focus errors should be compensated for.

One correction technique that can to some degree diminish these errors is the introduction of a plano-convex cylindrical lens in front of the beam shaper (Wetter 2001). The lens should be oriented with its axis perpendicular to the diodes slow divergence direction as shown in figure 4 . Rotating the lens by a small negative angle $\alpha$ around the $x$-axis causes a displacement of parts of the light sheet in the vertical direction. This translation in the positive y-direction depends upon the thickness of the traversed lens material and is therefore largest for that sub-beam that enters the lens at its center. The shift of the center relative to the border of the diodes light sheet is proportional to $\alpha$ and inversely proportional to the focal length of the cylindrical lens. It follows that for a given smile there is always an inclination angle that is capable of lifting the central part of the light sheet to the same height as its borders, provided that the focal length of the lens is short enough.

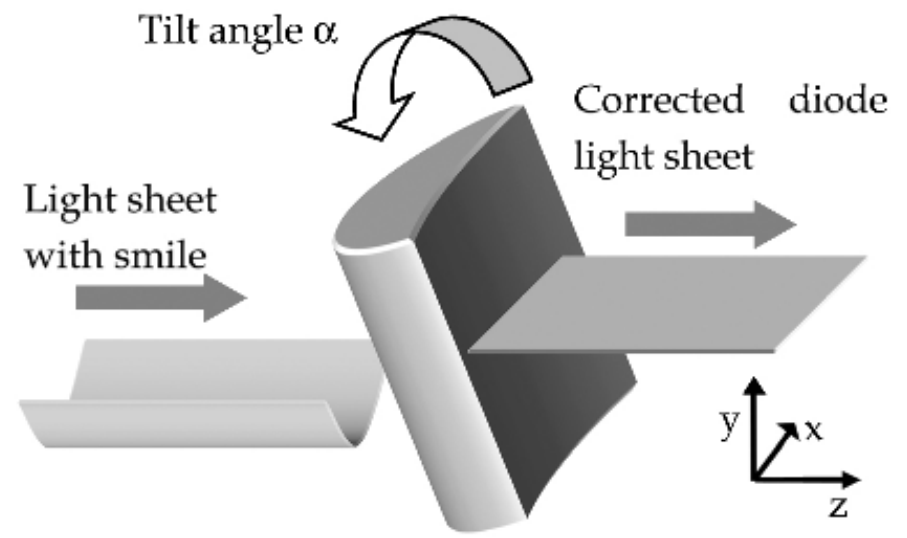

Fig. 4. Schematic diagram of the inclined cylindrical lens.

Given the cylindrical lens shape, this technique works best for a smile of parabolic curvature and with the center of the curvature in the middle of the light sheet. It has been shown that in such cases the height of the smile can be decreased by up to $68 \%$ reducing power loss due to clipping on mirror 1 by almost $27 \%$. Skew may be corrected in a similar way by tilting the lens slightly around its z-axis.

More complex systems use arrays of lenslets to control the divergence and beam propagation direction of the emitters in the bar. The number and spacing of the lenslets is such that they match the emitters of the bar. Each micro-lenslet has square dimensions 
and cylindrical shape in the horizontal and vertical direction in order to collimate the fast and slow axis at the same time achieving a divergence of less than 2 degrees in both directions. The focal length is very small, of the order of $100 \mu \mathrm{m}$, reducing greatly the size of the macroscopic optic of the beam shaper that follows. Smile reduces significantly the efficiency of such a system and therefore several lenslet arrays are mounted on top of each other with different row shapes that are described by polynomials which match different smile shapes. A lens array row shape can then be assigned to the specific smile of the diode bar (Hamilton et al., 2004). This technique allows the lenslets to be centered in front of each emitter and to carry out their function of beam collimation and aligning the beam into the right direction.

All of above mentioned beam-shaping techniques are intended to generate square beams of equal beam quality factors in both directions. However a square shape is not ideal for coupling into a fiber. To achieve better beam circularization one must change the individual emitter width. If the emitter in the middle of the bar is the largest and if the emitters become progressively smaller as one moves out to the last emitters on opposite ends, stacking as shown in figure 3 will provide for a round spot composed of stacked emitter beamlets.

\subsection{Brightness increase}

So far only beam circularization has been achieved. As stated by equation (1), brightness is inversely proportional to the area occupied by the source. In other words, high brightness is achieved with $100 \%$ fill factor and the "dead space" between the emitters of the bar has to be removed in order to increase the brightness. As shown earlier, this space cannot be eliminated at the source for cooling reasons and therefore it is necessary to eliminate it afterwards. This can be achieved for instance with the beam-shaper of figure 3 by adjusting the mirrors' positions and angles. In this figure, the light sheet emitted by the bar is sectioned into five sub-beams as also shown in figure 5 .
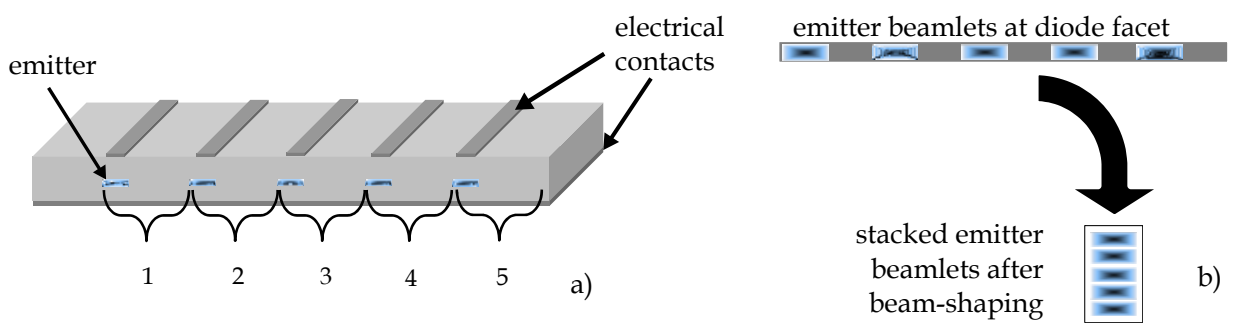

Fig. 5. a) Schematic of a diode bar with 5 emitters. The width of the brackets corresponds to the emitter spacing or emitter pitch. b) Schematic description of the "dead space" being removed by the beam-shaper.

Each sub-beam is composed of one part useful laser radiation and one part dead space. By moving mirror 2 in figure 3 along the negative $x$-direction the width of the sub-beam 1 will be decreased until only the emitter beamlet component from the sub-beam remains. If then 
the mirrors' spacing and the inclination angle are readjusted properly, all other four subbeams will undergo the same procedure and finally, only the 5 emitter beamlets will emerge from the beam-shaper, as shown in figure 5 .

Using the simple beam correction and shaping methods outlined in item 3, a power density of $46 \mathrm{~kW} / \mathrm{cm}^{2}$ can be achieved with standard diode bars accompanied by a loss of less than 20 percent in output power. The brightness achieved with this side-by-side beam combining techniques cannot be greater than the brightness of a single emitter because, at best, the power increases at the same rate as the area of the reconfigured beam.

A standard method to couple two diodes that have undergone beam-shaping is the polarization coupling with beam-splitters. In this method, diode 1 and diode 2 have orthogonal beam polarizations that are coupled by a special beam-splitter that is $100 \%$ transmissive for the polarization of diode 1 and reflects $100 \%$ of diode 2 at 45 degrees incidence, overlaying ideally both beams completely at the output, without loss of spectral brightness. The method is often used to increase the power launched into a fiber by a factor of two.

New developments in the area of facet coatings and passivation techniques have permitted power scaling using longer resonators and narrower emitters (with lower divergence). These bars have the same output power at only $25 \%$ of the total emitter facet area of the common $1 \mathrm{~cm}$ wide bars. They are called T-bars, super-bars or also mini-bars because their width is of the order of only $3 \mathrm{~mm}$. The reduced width not only decreases greatly the beam divergence and the quality factor in the slow axis direction but also increases its flatness and therefore decreases smile, skew and relative focus errors.

\subsection{Spectral beam combining}

Spectral beam combining, also called wavelength combining or incoherent beam combining, is a very efficient beam-shaping method that permits to overlap almost completely the beamlets of all emitters thereby increasing greatly the spatial brightness. The result is the same as if in figure $5 b$ ) only one beamlet would emerge from the beamshaper with the power of all 5 beamlets together and hence, the brightness increase is equal to the number of emitters. But this power increase comes at the price of reduced spectral brightness.

The working principle of spectral beam combining is to assign to each emitter a different wavelength with exclusive power-spectra that is then imaged by an optical system onto some wavelength-selective device capable of combining the different beamlets into one single output beam (Daneu et al., 2000). An example of such a device is shown in figure 6. In this particular beam-combining beam-shaper the front-facet of the diode bar is antireflection coated and hence, the resonator is formed between the bar's back-facet and the output mirror. The transform lens, the grating and the flat output coupler serve to assign to each emitter on the bar a different incidence angle on the grating and therefore a different center wavelength. The overall gain bandwidth of the diode and the dispersion of the grating must be adjusted such that the spectral emission bandwidth of each diode is exclusive. 


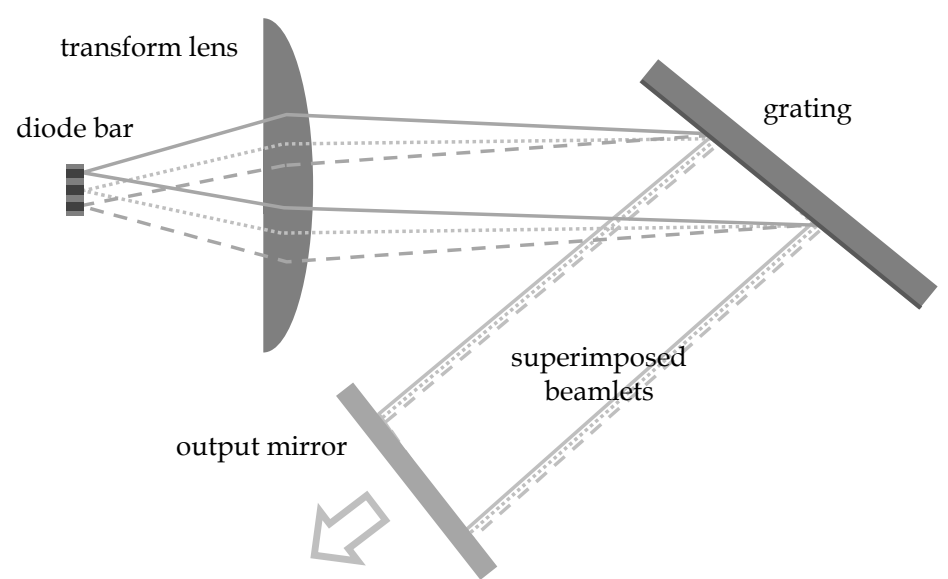

Fig. 6. Schematic of the working principle of beam-shaper of class "incoherent beam combiner".

The beam quality factors of the bar after beam-shaping achieved with above device are the same as of a single emitter, which is 10-20 in the slow axis direction and approximately 2 in the fast-axis direction. The measured brightness increase is about 20x, achieving close to 100 $\mathrm{MW} / \mathrm{cm}^{2} \mathrm{str}$.

It should be noted that this design is different from the well known wavelength-divisionmultiplexing (WDM) used to combine different wavelength in a waveguide in as much as this is a purely passive design whereas in WDM one generally tunes first each emitter independently and then combines them with the wavelength-selective device. The wavelength combination may occur also by means of prisms and volume-Bragg gratings (VBG) whilst for only a few beamlets one may also use dichroic mirrors.

One of the interesting features of this technique is that it is further power scalable because diodes can be stacked into a 2-dimensional array with much higher output power than a single bar and chirped VBGs can be used to assign individual wavelengths to the emitters which then are superposed by the grating (Chann et al., 2006).

\subsection{Coherent beam combining}

Coherent beam combining (CBC), also called coherent addition or phase-locked arrays, is a technique that permits joining of several beams increasing the spatial brightness. It differs from spectral beam combining in as much as there is no need to assign different wavelengths to each beamlet, however, the phase relation between the beamlets has to be well determined such that they all combine coherently in the output beam. As a result, this technique permits increase in spatial brightness and spectral brightness at the same time because the total bandwidth can be as small as the bandwidth of a single emitter. Because of this prospect, this class of beam-shaping has received a lot of attention. The method is very well known in microwave and radio-frequency applications as phased-array transmitters. 
However, this technique has so far not been able to give the expected results in laser applications, mainly because of the shorter wavelength, and it has been proven to be very challenging to maintain the phase relationships within a fraction of $2 \pi$ outside the laboratory.

The first step in coherent beam combining is to achieve beamlets that have the same polarization and the same phase and therefore may interfere coherently. Most methods used to obtain phase-locking of the beamlets require further that all beamlets operate at the same frequency, or single-frequency, although there are some important exceptions to this rule.

Two beams of equal power that travel side-by-side and interfere constructively already have a brightness increase of a factor two because the power doubles and the beam quality remains the same. This side-by-side combining (also called tiled-aperture combining or phased array) has been used since the 1970's mostly with semiconductor arrays whose emitters are closely spaced in a way that they optically couple through evanescent waves and excite some higher order transverse mode that incorporates all emitters (Scifres et al., 1978). Aside from this passive method of phase-locking (that may be applied also to fiber lasers) exist a series of active methods that generally result in a much more robust phase-lock amongst emitters (or channels). For example, one may use a periodic 2-D array of semiconductor emitters inside a Talbot cavity (a Talbot cavity images the near filed image of the array onto itself) and use a phase-plate (for example a spatial light modulator - SLM) between the array and the output coupling mirror that adjusts the optical path difference of each individual emitter. To calculate the phase introduced for each emitters' optical path sophisticated feedback-loops are required that may act upon a SLM or the emitter current or temperature to adjust the emitter's phase. If the emitters' resonator lengths are different and if there are several channels, as it is for example with combining of fiber lasers, it might become difficult to find longitudinal modes that are common to all resonators. In such cases one can use a single-frequency laser at the origin that becomes amplified in several separate channels and then apply active phase correction at the output. Up to 64 fibers have been combined in this manner (Bourderionnet et al., 2011).

In a next step one may further increase the spectral and spatial brightness by combining the separate channels into one single channel using for example diffraction gratings or beam splitters (this is called filled-aperture combining). A 50/50 beam-splitter normally has two outputs unless both beams are adjusted in phase such that one output interferes destructively. The correct path difference may be obtained actively or passively by careful positioning and calculation of the beam splitters' dimensions as shown in figure 7. An additional benefit of this scheme is that because all beamlets share the same resonator at the output coupler, there is generally no need for active phase control at the output end. A total of 16 beamlets originating from the same laser rod have been added in this manner generating an overall spectral brightness increase of thirty times (Eckhouse et al., 2006).

There is also a class of beam CBC methods that, instead of comprising arrays or beam splitters, use non-linear effects such as non-linear mirrors based on second harmonic generation (SHG) or Brillouin scattering. 


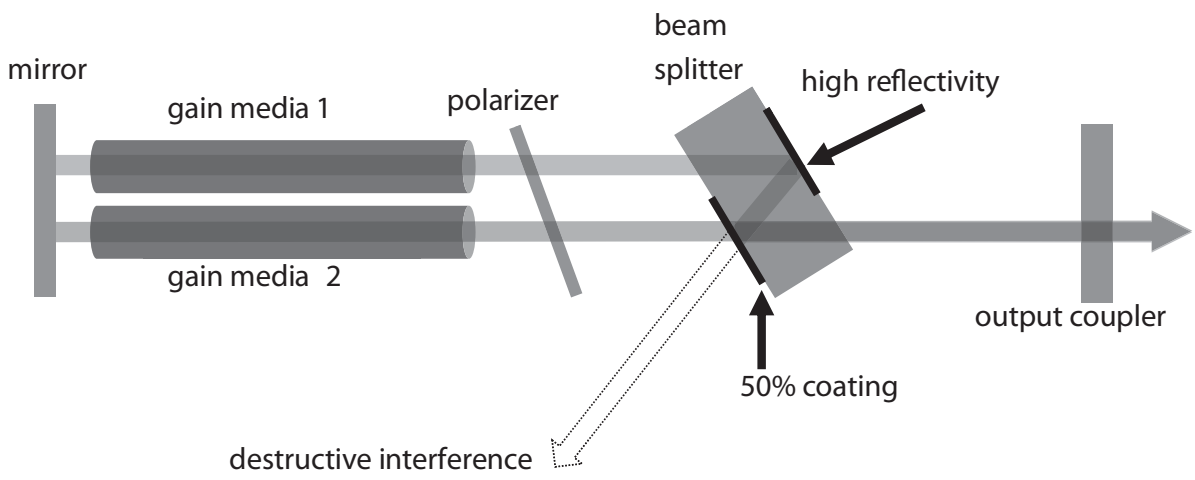

Fig. 7. Schematic of two channels being coherently added by means of a beam splitter.

\section{Spectral narrowing}

Many applications would benefit from the advantages of diode lasers mentioned in this chapter, if the linewidth were smaller than the $3 \mathrm{~nm}$ (approximately half a $\mathrm{THz}$ ) of standard high-power diode arrays. LIDAR detection techniques used for trace gas detection and wind speed measurements, optical pumping of rare-earth solid state-lasers and mm-wave lasers, laser cooling for atomic clocks and WDM in optical communication systems are all applications that could use much smaller linewidth. These diverse applications have been traditionally served by tunable broad emission bandwidth lasers such as Ti:Sapphire and Dye lasers.

The high power diode lasers (HPDLs) can be easily tuned with external cavities (as already seen in the former chapter) and depending on cavity parameters and reflectivity of the emitter facets' AR coating, the tuning range is naturally around $30 \mathrm{~nm}$ even at very high output powers. It therefore represents in principle an ideal substitute for the Ti:Sapphire or dye laser if the bandwidth can be made small enough as required by the application.

Several injection schemes have been tested nowadays and they can be divided into selfinjection locking and master-injection locking. The former usually requires an external cavity that supplies frequency-selective feedback and the latter relies usually on a singlefrequency master oscillator. In self-injection schemes the frequency-selective device for the feedback from the external cavity comes normally from a diffraction grating but also a VBG, an etalon or a prism can serve to this purpose.

The most common external cavity schemes employing gratings are the well known Littrow configuration, which directly injects the first diffraction order back to the diode, and the Littman-Metcalf configuration, which uses an additional mirror to reflect the first order back and therefore passing the beam twice through the grating. The advantage of the former method is higher output power (throughput of the system is typically $80 \%$ ) whereas the latter method shows generally more spectral narrowing because of the double pass through the grating and also a fixed output beam direction. 
Narrowing can only be achieved if the beamlets from each emitter overlap at the grating (Wetter, 2007). It is therefore essential that smile is minimized because in the end it will increase the linewidth by approximately $0.9 \mathrm{~nm}$ per $\mu \mathrm{m}$ of smile. But overlap can be increased if a telescope is used in the beam path of magnification $\mathrm{M}$. The better overlap at the grating of the magnified images will reduce angular spread and decrease the influence of smile by a factor of $1 / \mathrm{M}$ and hence further narrow the diodes' spectra. Additionally the light re-injected from the external cavity will match better each emitter's position and therefore less re-injected power is necessary to force the emitters to oscillate at the desired wavelength. The amount of light that gets re-injected can be adjusted for example with a half-wave plate.

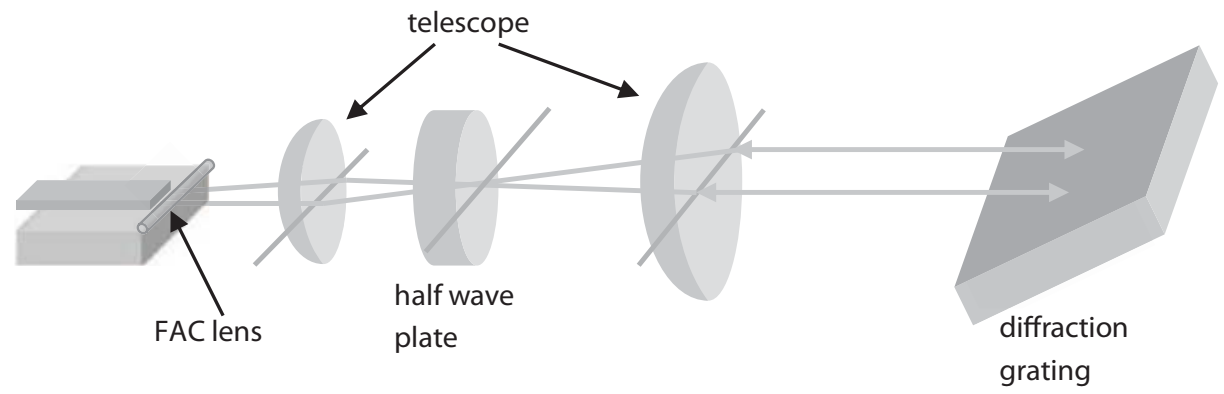

Fig. 8. Schematic of a Littrow configuration using an intracavity telescope and a half-wave plate to adjust the retro-injected power.

Wavelength narrowing down to less than $40 \mathrm{pm}$, corresponding to a linewidth of about 10 $\mathrm{GHz}$ at NIR wavelength, is achievable using VGBs and Littrow set-ups, respectively (Zhdanov et al., 2007) (Vijayakumar et al., 2011) , and spectral brightness increase of up to 86 times has been obtained using HPDL with up to 21 emitters. This is enough narrow for optically pumping alkali vapor lasers such as potassium vapor and cesium.

The self-injection narrowing shown in figure 8 is proportional to the number of emitters and better results of spectral brightness increase are achieved with fewer emitters because the overall width is smaller and therefore smile can be reduced. Even with new production methods, smile continues at about $1 \mu \mathrm{m}$ height. Increasing the magnification augments the size and decreases the mechanical stability of the set-up. It seems therefore, that the most promising approach so far is to use BALs of short cavity width. As was shown earlier, these broad area diode lasers can have quite small width and present very high output powers. Using a $1000 \mathrm{~mm}$ wide BAL with maximum output power of up to $25 \mathrm{~W}$ in a LittmanMetcalf configuration, linewidth narrowing from $2 \mathrm{~nm}$ down to $1.8 \mathrm{MHz}$ has been achieved at output powers up to $10 \mathrm{~W}$ (Sell et al., 2009). This very small linewidth is not frequency stable and a measurement over 0.5 seconds results in a linewidth of $130 \mathrm{MHz}$, which shows the influence of temperature and mechanical instabilities.

\section{Conclusions}

Standard HPDL bars are nowadays factory equipped with beam-shapers for beam circularization with only a minor increase in cost. This kind of beam-shaper generates a circular beam of equal quality factors in the horizontal and vertical direction which can be 
used to achieve, for example, very efficient fiber-coupling. Up to $60 \mathrm{~W}$ of output power from fibers of $100 \mu \mathrm{m}$ fiber core diameter and NA of 0.22 are standard using a single bar. Polarization coupling of two bars into the same fiber diameter with total output power of $120 \mathrm{~W}$ is also commercially standard. Systems using spectral and coherent beam combining are still relatively expensive but are bound to become important players on the market of high-power, high-brightness lasers. Better facet passivation techniques have also boosted lately the competition between BALs and diode bars. Broad area diodes have a series of advantages such as better spatial and spectral brightness as outlined in this chapter and if powers should become comparable to diode HPDL arrays then they are certainly advantageous for many applications.

\section{References}

Bourderionnet, J., Bellanger, C., Primot, J. \& Brignon, A. 2011. Collective coherent phase combining of 64 fibers. Optics Express, 19, 17053-17058.

Chann, B., Goyal, A. K., Fan, T. Y., Sanchez-Rubio, A., Volodin, B. L. \& Ban, V. S. 2006. Efficient, high-brightness wavelength-beam-combined commercial off-the-shelf diode stacks achieved by use of a wavelength-chirped volume Bragg grating. Optics Letters, 31, 1253-1255.

Crump, P., Blume, G., Paschke, K., Staske, R., Pietrzak, A., Zeimer, U., Einfeldt, S., Ginolas, A., Bugge, F., Hausler, K., Ressel, P., Wenzel, H. \& Erbert, G. 20W continuous wave reliable operation of $980 \mathrm{~nm}$ broad-area single emitter diode lasers with an aperture of $96 \mathrm{mu}$. Conference on High-Power Diode Laser Technology and Applications VII, Jan 26-27 2009 San Jose, CA. Spie-Int Soc Optical Engineering.

Daneu, V., Sanchez, A., Fan, T. Y., Choi, H. K., Turner, G. W. \& Cook, C. C. 2000. Spectral beam combining of a broad-stripe diode laser array in an external cavity. Optics Letters, 25, 405-407.

Eckhouse, V., Ishaaya, A. A., Shimshi, L., Davidson, N. \& Friesem, A. A. 2006. Intracavity coherent addition of 16 laser distributions. Optics Letters, 31, 350-352.

Hamilton, C., Tidwell, S., Meekhof, D., Seamans, J., Gitkind, N. \& Lowenthal, D. High power laser source with spectrally beam combined diode laser bars. Conference on High-Power Diode Laser Technology and Applications II, Jan 26-27 2004 San Jose, CA. 1-10.

Knigge, A., Erbert, G., Jonsson, J., Pittroff, W., Staske, R., Sumpf, B., Weyers, M. \& Trankle, G. 2005. Passively cooled $940 \mathrm{~nm}$ laser bars with $73 \%$ wall-plug efficiency at $70 \mathrm{~W}$ and 25 degrees C. Electronics Letters, 41, 250-251.

Muller, A., Vijayakumar, D., Jensen, O. B., Hasler, K.-H., Sumpf, B., Erbert, G., Andersen, P. E. \& Petersen, P. M. 2011. 16 W output power by high-efficient spectral beam combining of DBR-tapered diode lasers. Optics Express, 19, 1228-1235.

Scifres, D. R., Burnham, R. D. \& Streifer, W. 1978. Phase-Locked Semiconductor-Laser Array. Applied Physics Letters, 33, 1015-1017.

Sell, J. F., Miller, W., Wright, D., Zhdanov, B. V. \& Knize, R. J. 2009. Frequency narrowing of a $25 \mathrm{~W}$ broad area diode laser. Applied Physics Letters, 94, 51115 - 51115-3.

Vijayakumar, D., Jensen, O. B., Barrientos-Barria, J., Paboeuf, D., Lucas-Leclin, G., Thestrup, B. \& Petersen, P. M. 2011. Narrow line width operation of a $980 \mathrm{~nm}$ gain guided tapered diode laser bar. Optics Express, 19, 1131-1137. 
Wenzel, H., E. Bugge, M. Dallmer, F. Dittmar, J. Fricke, K. H. Hasler, and G. Erbert. 2008. Fundamental-lateral mode stabilized high-power ridge-waveguide lasers with a low beam divergence. Ieee Photonics Technology Letters, 20, 214-216.

Wetter, N. U. 2001. Three-fold effective brightness increase of laser diode bar emission by assessment and correction of diode array curvature. Optics and Laser Technology, 33, 181-187.

Wetter, N. U. 2007. Tunable dual wavelength emission and bandwidth narrowing of a laser diode array with a simple external cavity. Applied Physics B-Lasers and Optics, 86, 515-518.

Zhdanov, B. V., Ehrenreich, T. \& Knize, R. J. 2007. Narrowband external cavity laser diode array. Electronics Letters, 43, 221-222. 


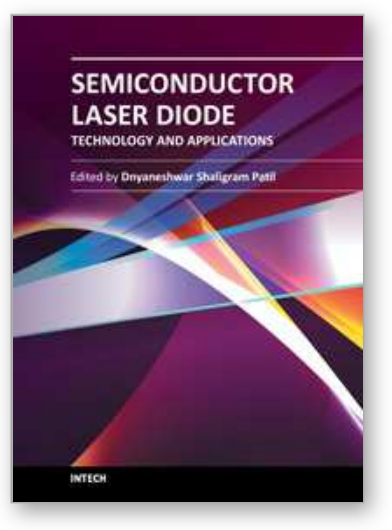

\author{
Semiconductor Laser Diode Technology and Applications \\ Edited by Dr. Dnyaneshwar Shaligram Patil
}

ISBN 978-953-51-0549-7

Hard cover, 376 pages

Publisher InTech

Published online 25, April, 2012

Published in print edition April, 2012

This book represents a unique collection of the latest developments in the rapidly developing world of semiconductor laser diode technology and applications. An international group of distinguished contributors have covered particular aspects and the book includes optimization of semiconductor laser diode parameters for fascinating applications. This collection of chapters will be of considerable interest to engineers, scientists, technologists and physicists working in research and development in the field of semiconductor laser diode, as well as to young researchers who are at the beginning of their career.

\title{
How to reference
}

In order to correctly reference this scholarly work, feel free to copy and paste the following:

Niklaus Ursus Wetter (2012). Spectral Narrowing and Brightness Increase in High Power Laser Diode Arrays, Semiconductor Laser Diode Technology and Applications, Dr. Dnyaneshwar Shaligram Patil (Ed.), ISBN: 978953-51-0549-7, InTech, Available from: http://www.intechopen.com/books/semiconductor-laser-diodetechnology-and-applications/spectral-narrowing-and-brightness-increase-in-high-power-laser-diode-arrays

\section{INTECH}

open science | open minds

\section{InTech Europe}

University Campus STeP Ri

Slavka Krautzeka 83/A

51000 Rijeka, Croatia

Phone: +385 (51) 770447

Fax: +385 (51) 686166

www.intechopen.com

\section{InTech China}

Unit 405, Office Block, Hotel Equatorial Shanghai

No.65, Yan An Road (West), Shanghai, 200040, China

中国上海市延安西路65号上海国际贵都大饭店办公楼 405 单元

Phone: +86-21-62489820

Fax: $+86-21-62489821$ 
(C) 2012 The Author(s). Licensee IntechOpen. This is an open access article distributed under the terms of the Creative Commons Attribution 3.0 License, which permits unrestricted use, distribution, and reproduction in any medium, provided the original work is properly cited. 